\title{
Dynamic model of whole cortex reveals disassortative hub structure in the intracortical connectome
}

\author{
Matthieu Gilson ${ }^{1 *}$, Ruben Moreno-Bote ${ }^{2,3}$, Adrian Ponce-Alvarez ${ }^{1}$, Petra Ritter ${ }^{4}$, Gustavo Deco ${ }^{1}$ \\ From 24th Annual Computational Neuroscience Meeting: CNS*2015 \\ Prague, Czech Republic. 18-23 July 2015
}

The brain exhibits complex spatio-temporal patterns of activity. In particular, its baseline neural activity for idle subjects or animals has a specific structure, also called functional connectivity (FC): cortical areas experience correlated fluctuations at rest as revealed by imaging techniques (e.g., fMRI, EEG and MEG). Moreover, these correlation patterns become more expressed or suppressed depending on the engaged task. The putative function for this stereotypical background activity has been actively questioned by the neuroscience community. A Bayesian hypothesis is that it reflects the variety of possible coactivations of cortical areas for usual tasks, forming a prior representation of the environment and interactions wherewith.

The present study relies on our recently developed model [1] in which noise diffusion is constrained by intracortical white-matter projections to reproduce FC observed in experimental data (fMRI BOLD signal). In this model, noise has a functional role and represents the variability of neural activity during tasks. This model allows us to explore the role of various parameters in shaping FC, such as the local nonlinear dynamics of cortical areas. More importantly, we can infer the efficacies of intracortical projections, also called effective connectivity (EC), from experimental data. EC plays a crucial role in shaping $\mathrm{FC}$ and differs from the structural connectivity (SC) that is typically obtained using diffusion tensor imaging (DTI), which estimates the density of white-matter fibers. It is important to notice that the efficacies of these fibers may differ from their density, due to the concentration of synapses formed at their

\footnotetext{
* Correspondence: matthieu.gilson@upf.edu

'Center for Brain Cognition, Universitat Pompeu Fabra, 08018, Barcelona, Spain

Full list of author information is available at the end of the article
}

end, the type of neurotransmitters associated and the excitability of target neural populations. In the end, our model combines anatomical SC and activity FC to evaluate what drives the neural dynamics, embodied in EC.

Practically, directed EC can be estimated using two matrices of empirical FC, namely FC0 for correlations with zero time shift and $\mathrm{FC} \tau$ for correlations with a given time shift $\tau>0$. We apply our method to infer the intracortical EC from experimental fMRI data that consists of $\sim 20$ minutes of recording for 25 idle subjects. The model reproduces both empirical FC0 and FC $\tau$ matrices with a Pearson correlation coefficient of 0.65 . The recovered EC connectivity is significantly asymmetric, with a value of 0.3 for an index that scales from 0 for symmetric matrices to 1 for asymmetric matrices. Moreover, EC exhibits hubs that have either strong incoming or outgoing weights, but not both. This disassortative property is expected to exert a strong influence in shaping the whole network dynamics.

\section{Acknowledgements \\ MG and GD were supported by the Human Brain Project. RM-B was supported by the Ramon y Cajal Spanish Award (RYC-2010-05952), the Marie Curie FP7-PEOPLE-2010-IRG grant (PIRG08-GA-2010-276795), and the Programa Estatal de Investigacion Cientifica y Tecnica de Excelencia (grant PSI2013-44811-P). PR acknowledges the support of the James S. McDonnel Foundation (Brain Network Recovery Group JSMF22002082), the German Ministry of Education and Research (Bernstein Focus State Dependencies of Learning 01GQ0971) and the Max-Planck Society (Minerva Program).}

\begin{abstract}
Authors' details
${ }^{1}$ Center for Brain Cognition, Universitat Pompeu Fabra, 08018, Barcelona, Spain. ${ }^{2}$ Parc Sanitari Sant Joan de Déu and Universitat de Barcelona, Esplugues de Llobregat, 08950, Barcelona, Spain. ${ }^{3}$ CIBERSAM, Esplugues de Llobregat, 08950 Barcelona, Spain. ${ }^{4}$ Bernstein Center for Computational Neuroscience, Berlin, 10117, Germany.
\end{abstract}

Published: 18 December 2015 


\section{Reference}

1. Deco G, Ponce-Alvarez A, Hagmann P, Romani G, Mantini D, et al: How local excitation-inhibition ratio impacts the whole brain dynamics. J Neurosci 2014, 34:7886-7898.

doi:10.1186/1471-2202-16-S1-P57

Cite this article as: Gilson et al:: Dynamic model of whole cortex reveals disassortative hub structure in the intracortical connectome. BMC

Neuroscience 2015 16(Suppl 1):P57.

Submit your next manuscript to BioMed Central and take full advantage of:

- Convenient online submission

- Thorough peer review

- No space constraints or color figure charges

- Immediate publication on acceptance

- Inclusion in PubMed, CAS, Scopus and Google Scholar

- Research which is freely available for redistribution

Submit your manuscript at www.biomedcentral.com/submit

() Biomed Central 